\title{
Explotación de la pesquería de arrastre de camarón durante el período 1991-1999 en el Golfo de Nicoya, Costa Rica
}

Farid A. Tabash Blanco

Estación de Biología Marina, Escuela de Ciencias Biológicas, Universidad Nacional, Apartado Postal 86-3000, Puntarenas, Costa Rica; atabash@una.ac.cr

Recibido 28-X-2005. Corregido 10-VII-2006. Aceptado 15-XII-2006.

\begin{abstract}
Exploitation of the shrimp trawl fishery in the period 1991-1999 at the Gulf of Nicoya, Costa Rica. In Costa Rica, the Gulf of Nicoya shrimp fishery originated in 1952 and represented one of the most important economic activities in the region. Nevertheless, overfishery reduced the captured volumes to levels that prevent this commercial activity. I analyzed official fishery statistics between 1991 and 1999. These species involved are: two species of white shrimp, (Litopenaeus occidentalis and L. stylirostris), the "titi" shrimp (Xiphopenaeus riveti), the brown shrimp (Farfantepenaeus californiensis), the "pinki" shrimp (F. brevirostris) and the "fidel" shrimp (Solenocera agassizi). All the species reached the Maximum Sustainable Yield in the decades of 1970 and 1980 and are now found at over-exploitation levels. I recommend that this shrimp trawl fishery be completely closed down. Rev. Biol. Trop. 55 (1): 207-218. Epub 2007 March. 31.
\end{abstract}

Key words: shrimp, fishery, Nicoya Gulf, assessment, colapse, over-exploitation.

En Costa Rica la pesca del camarón se concentra en las aguas del litoral Pacífico, principalmente en el Golfo de Nicoya, donde se explota el camarón blanco (Vitola 1985). El Golfo de Nicoya, por sus características ambientales, es considerado uno de los estuarios tropicales más productivos, posee un área cercana a los $1500 \mathrm{Km}^{2}$, constituido en su mayor parte por fondo lodoso y arenoso, con profundidades entre 5-200 $\mathrm{m}$ y bordeado por bosques de manglar (Martínez 1988).

Actualmente la captura de camarón por parte de la flota arrastrera en el Golfo de Nicoya se compone de dos especies de camarón blanco (Litopenaeus occidentalis Streets, 1871 y L. stylirostris Stimpson, 1871), del camarón tití (Xiphopenaeus riveti Bouvier, 1907), del camarón café (Farfantepenaeus californiensis Holmes, 1900), del camarón "pinki" (F .brevirostris Kingsley, 1878) y del fidel (Solenocera agassizi Burkenroad, 1938) (Palacios 2003).
La pesca semi-industrial del camarón inició a principios de la década de los años 50 cuando se introdujo la red de arrastre para la captura del camarón (López 1968). Los primeros datos oficiales de desembarques de camarón se refieren al año 1952, en el que se registraron 43.2 ton. Hasta mediados de la década de 1950, el camarón blanco era la única especie de interés, ya que las otras especies capturadas se devolvían al mar (Martínez 1988).

Hasta 1952 la captura en el Golfo estaba compuesta solo por camarón blanco, el cual en aquella época incluía también a la especie L. vannamei, que desaparece de las pesquerías a principios de la década de los 80. A partir de 1956, el camarón tití ya es incluido en la pesquería. Luego en 1973 se incluye también al camarón "pinki" y al café. Finalmente, en 1978 el fidel es incluido. Para 1999, la pesca de camarón se distribuyó de la siguiente manera: camarón blanco $23 \%$, tití $38 \%$, café $1 \%$, rosado $18 \%$ y fidel $20 \%$ (INCOPESCA 1999). 
Entre 1985 y 1986 las capturas de camarón registraron un máximo de 4300 ton métricas (TM) entrando luego en un proceso de disminución en las capturas, hasta alcanzar en 1998 una captura total de 1304.2 TM (INCOPESCA 1999). El propósito de este trabajo es ofrecer un panorama de la evolución de la pesca de las principales especies de camarón que fueron capturadas por la flota arrastrera en el Golfo de Nicoya durante la última década del siglo XX, así como analizar esta pesquería utilizando para ello modelos pesqueros analíticos de evaluación directa, con el objeto de que esta información sea de utilidad en la toma de decisiones para tratar de ordenar esta actividad.

\section{MATERIALES Y MÉTODOS}

Una serie de tiempo de 10 años (19911999) de estadísticas de captura básicas para cada una de las especies que componen el recurso camarón, obtenidas para la flota pesquera de arrastre que opera en el Golfo de Nicoya, es analizada en detalle en el presente trabajo. Para el año 1999, solo se cuenta con datos de dos meses, sin embargo se decidió incluir esta información con el objeto de comparar la relación captura-esfuerzo. En relación conl esfuerzo anual aplicado se estima el número de días pesca registrados por el total de barcos.

Las herramientas analíticas empleadas se basan en los modelos introducidos por Schaefer (1954, 1957), así como las correspondientes revisiones al modelo realizadas por Fox (1970), Ricker (1975), Gulland (1983), Pauly (1984) y Sparre y Venema (1992). La derivación de CPUE y su transformación logarítmica fueron utilizadas para el desarrollo de los modelos de producción. Adaptando también el método de "filo de navaja" (Mostella y Tukey 1977, Tukey 1977) presentado en Pauly (1984). Donde $\mathrm{MSY}_{1}$ representa el Máximo Rendimiento Sostenible (MRS) obtenido usando todos los pares de datos disponibles dentro de las estadísticas de pesca de las poblaciones de camarón blanco.
Con ello se pretende conocer el estado actual del camarón blanco como recurso natural y su tendencia en tiempo y espacio; con el objetivo de estimar el esfuerzo pesquero óptimo a que puede someterse la población de camarón blanco del Golfo de Nicoya. El modelo representa la biomasa en función del esfuerzo pesquero al que está sometida una población. La ecuación contempla diversos parámetros poblacionales del camarón blanco como: tasa de crecimiento intrínseca de la población, capacidad de carga del medio y coeficiente de capturabilidad (proporción de la población disponible de ser capturada).

Con el propósito de obtener una visión más amplia y realista de la evolución de la pesca del camarón blanco, se incluyó información proveniente de la base de datos histórica, que comprende los períodos desde 1969 a 1984 y de 1991 a 1998 en la aplicación del modelo de producción de Schaefer.

\section{RESULTADOS}

Los datos para la aplicación de los modelos analíticos desarrollados para la pesca del camarón café y del camarón rosado se presentan en las Figuras 1 y 2, respectivamente. Los resultados estadísticos del análisis de regresión lineal y los estimados de Máximo Rendimiento Sostenido obtenidos a través de los Modelos de Producción Excedentaria de Schaefer y Fox para las pesquerías de camarón rosado y fidel, se presentan en el Cuadro 1.

Al considerar la aplicación de estos modelos, la hipótesis de base supone que a cada estado de la población de camarones caracterizado por una biomasa dada, le corresponde un equilibrio estable con el medio. Este equilibrio es dinámico, los individuos que se reclutan, los incrementos relativos en peso y eventualmente las inmigraciones, compensan los efectos de mortalidad natural, por pesca y las emigraciones.

En las poblaciones de camarón rosado y café, la presión de pesca es el primer factor que condiciona la abundancia de la población, 


\section{CUADRO 1}

Estimados de Rendimiento Máximo Sostenido (RMS) y de esfuerzo óptimo a aplicar en las pesquerías del camarón rosado y fidel (F. brevirostris y S. agassizi) en el Golfo de Nicoya, Costa Rica

\section{TABLE 1}

Maximum Sustainable Yield (MSY) and optimum effort to apply in the pink and fidel shrimp (F. brevirostris and S. agassizi) fisheries in the Nicoya Gulf, Costa Rica

Valores estimados

Parámetros

Camarón rosado

camarón fidel

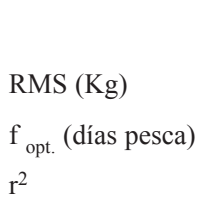

$\mathrm{r}^{2}$

\begin{abstract}
Schaefer
\end{abstract}
Fox

252714.8

2284

0.67
Schaefer

127094.45

2169.8

0.74
Fox

132547.2

2302

0.72

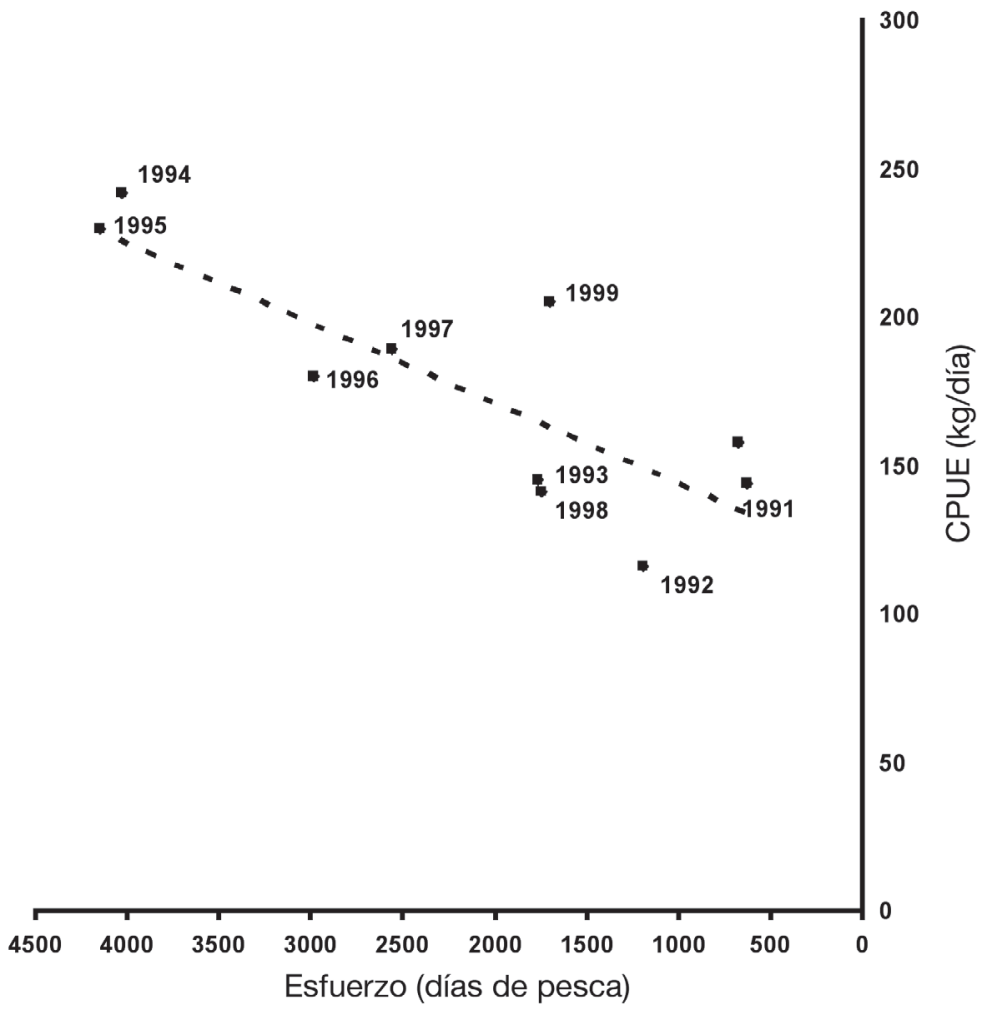

Fig. 1. Regresión lineal (modelo de Schaefer), para la pesca de arrastre del camarón café F. californiensis, en el Golfo de Nicoya, Costa Rica, 1991-1999.

Fig. 1. Linear regression (Schaefer model) for the trawl fishery of brown shrimp F. californiensis, in the Gulf of Nicoya, Costa Rica, 1991-1999. 


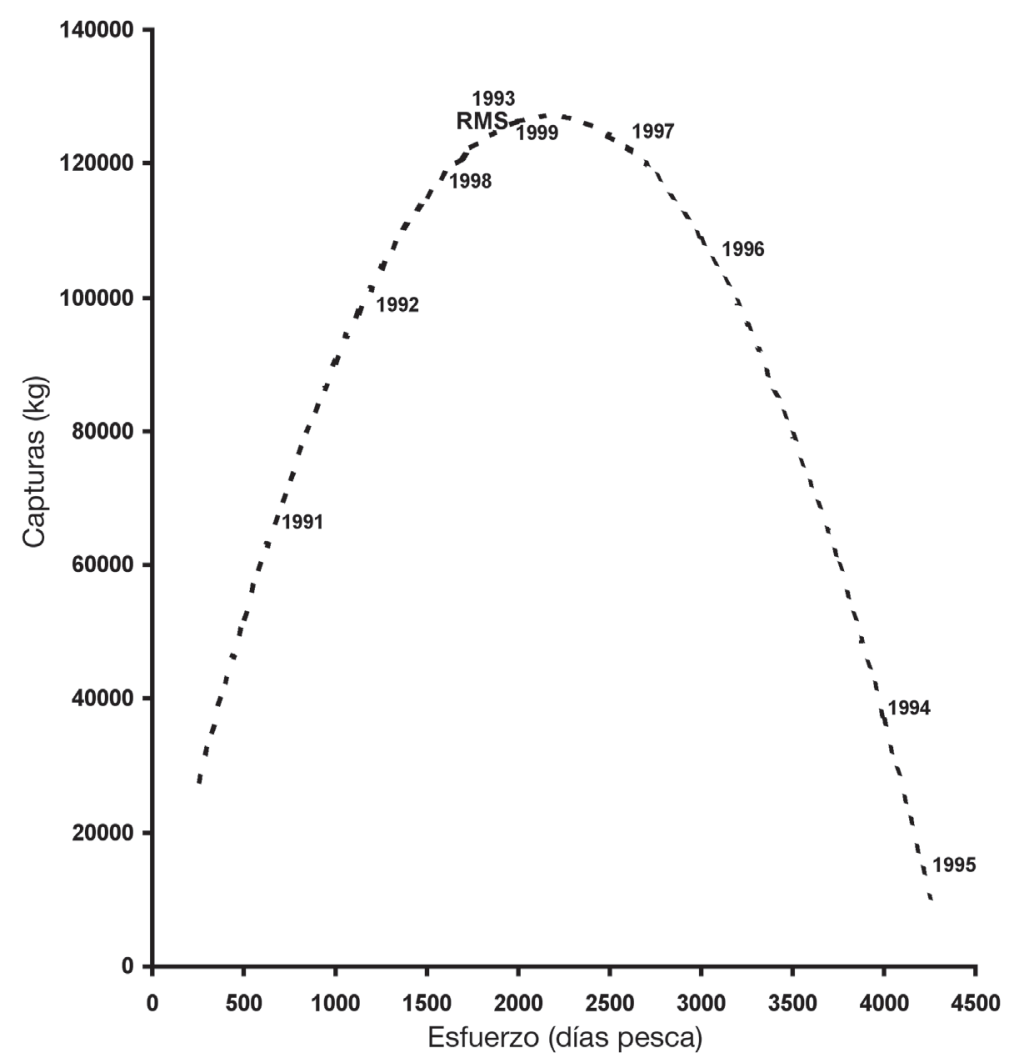

Fig. 2. Curva de Schaefer para la estimación del Rendimiento Máximo Sostenido (RMS) en la pesquería de arrastre del camarón fidel (S. agassizi), en el Golfo de Nicoya, Costa Rica.

Fig. 2. Schaefer curve for Maximum Sustainable Yield (MSY) estimation in the trawl fishery of fidel shrimp (S. agassizi), Gulf of Nicoya, Costa Rica.

se supone que a un nivel de esfuerzo dado la población alcanzará un estado donde, en promedio, no cambiará ni su dimensión ni sus características.

La pesca de camarón café presenta un incremento desmedido en el esfuerzo entre 1994 y 1995, casi un $350 \%$; sin embargo este incremento no se refleja en la capturas (96 kgdía $^{-1}$ ), en relación con el promedio registrado para el período 1991-1993 (140 kgdía $\left.{ }^{-1}\right)$. Es evidente que la relación esfuerzo-captura, representa el efecto de capturas más allá del Rendimiento Máximo Sostenido, lo que probablemente da paso a una sobreexplotación biológica (Fig. 1).

Para el camarón Fidel, el RMS estimado fue de $127094.45 \mathrm{kgdía}^{-1}$, producto de un esfuerzo óptimo de 2169.8 días de pesca, el cuál en la década del 90 fue sobrepasado desde 1993, de ahí que se estime la sobrepesca del camarón fidel cerca de un $165 \%$ (Fig. 2).

La captura de camarón fidel ha presentado desde 1995, una disminución no sostenida en los niveles de desembarque. Probablemente producto del excesivo esfuerzo de pesca aplicado, ello provoca que el daño a la población desovante sea muy grande. El comportamiento de los desembarques de camarón fidel así lo demuestran, tal y como se observa en la curva polinomial (Fig. 3).

Otra evidencia de lo afirmado, se presenta al analizar la evolución de las capturas del camarón rosado $F$. brevirostris, en el período 1996-1997, las capturas se mantuvieron al 


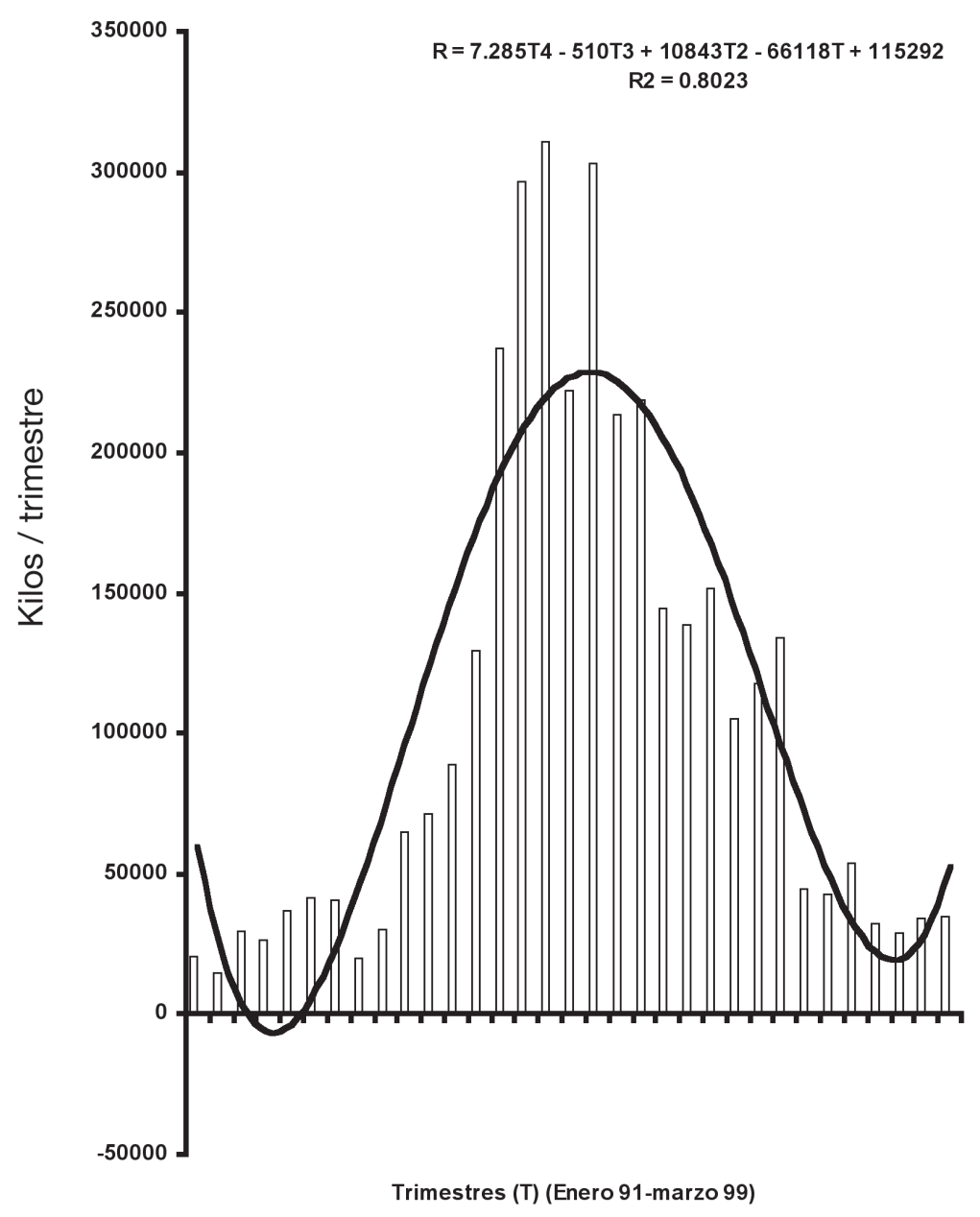

Fig. 3. Variación trimestral (T) en el rendimiento (R) de los desembarques del camarón fidel (S. agassizi) capturados por la flota de arrastre en el Golfo de Nicoya, Costa Rica.

Fig. 3. Quarterly variation (T) in the yield (R) of fidel shrimp (S. agassizi) landings by the trawling fleet in the Gulf of Nicoya, Costa Rica.

mismo nivel del bienio 95-96, pero ello producto de un incremento de $600 \mathrm{~h}$ en el esfuerzo de pesca (Fig. 4).

De acuerdo con la evolución anual de las capturas desde 1991 hasta 1999, se observa que los incrementos en los niveles de captura son el producto de un incremento en los niveles de esfuerzo. Para el período 1997-1998, el incremento en el nivel de esfuerzo pesquero ha sido exponencial mientras que las capturas tienden a disminuir. Es muy probable que al aumentar el esfuerzo no solo se haya impedido que esta pesquería tuviese una oportunidad para estabilizarse, sino que más bien se acelero el proceso de desequilibrio.

Para el camarón rosado, $F$. brevirostris se estimo un RMS de 252422.56 kgdía $^{-1}$ que corresponde a un esfuerzo óptimo de 2284 días de pesca. Esta especie para la década del 90 ya había sobrepasado su RMS. En 1995, el esfuerzo aplicado se estimó en 2200 días de pesca, pero probablemente porque el recurso ya no justificaba un esfuerzo mayor, ello queda evidenciado al observar una disminución en 


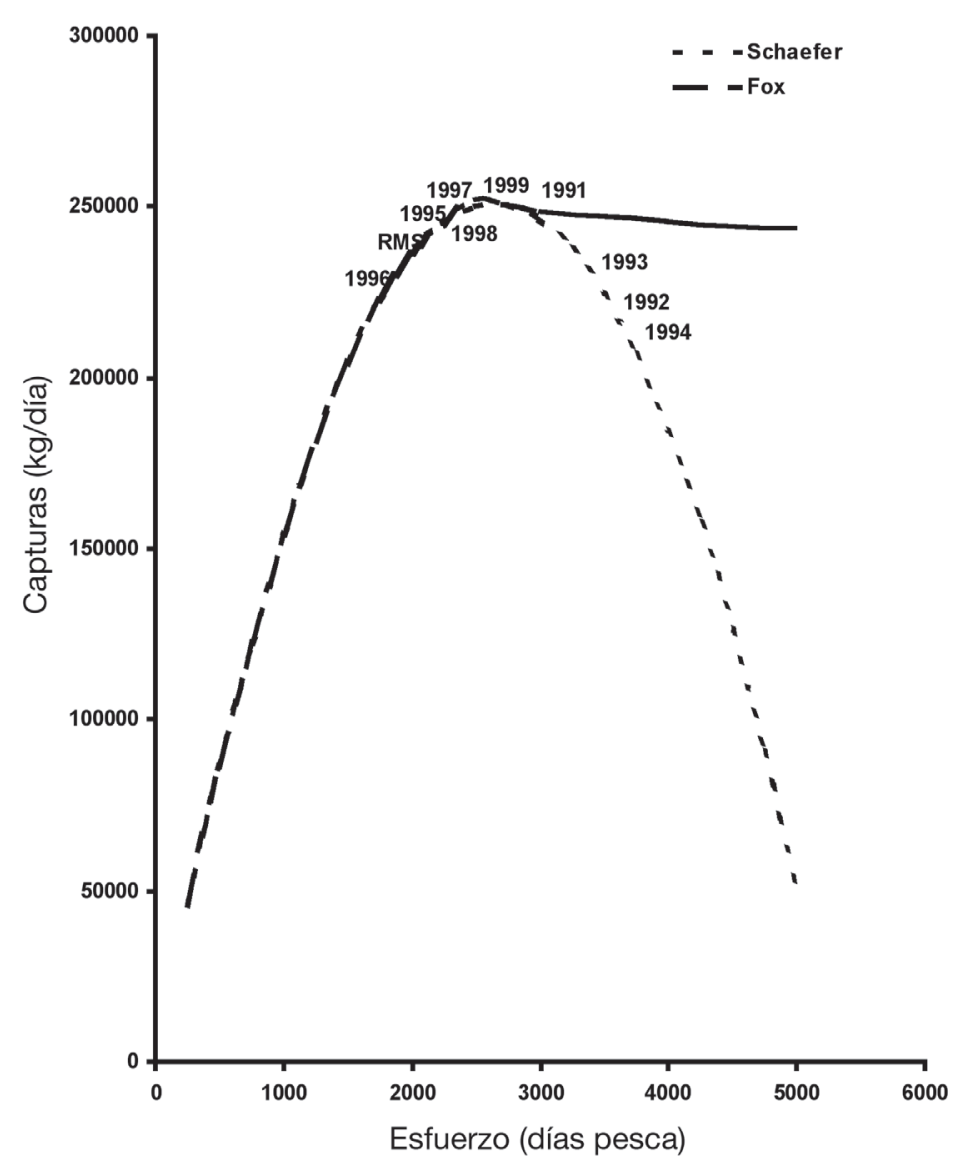

Fig. 4. Curva de Schaefer y Fox, con la evolución anual de la pesca de arrastre del camarón rosado (F. brevirostris), en relación con el estimado de Máximo Rendimiento Sostenido (MRS), en el Golfo de Nicoya, Costa Rica.

Fig. 4. Schaefer and Fox curve, with the annual evolution of pink shrimp trawl fishery (F. brevirostris) in relation with the Maximum Sustainable Yield (MSY) estimated in the Gulf of Nicoya, Costa Rica.

las capturas de $229820 \mathrm{~kg}$ o sea de un $45 \%$ menos, para el período 1990-1995.

En la Figura 5 se observa como en la década del 90 se presentó un incremento en los volúmenes de camarón blanco entregados a los centros de acopio por la flota semi-industrial como consecuencia del aumento en los niveles de esfuerzo pesquero. Los picos de captura, durante lo que podríamos llamar "buenos años", se mantuvieron constantes; sin embargo el esfuerzo dirigido hacia esta especie se ha duplicado entre un año y otro a partir de 1994, provocando como consecuencia lógica, bajos valores en los estimados de CPUE (la captura se ha mantenido en niveles más bajos que el esfuerzo correspondiente), y con fluctuaciones de corto plazo de gran magnitud.

El aspecto más importante a considerar en el análisis de estas curvas eumétricas es que no existe un máximo de producción que ascienda continuamente hasta un valor de producción asintótica. Esto significa que en 1986 al empezar la curva a descender, la pesquería del camarón blanco entra en un proceso que solo puede ser detenido haciendo que $\mathrm{F}=0$ (Herrington 1943, Ricker 1975).

El RMS y el esfuerzo óptimo aplicado $\left(f_{\text {RMS }}\right)$ estimados por los métodos de Schaefer 


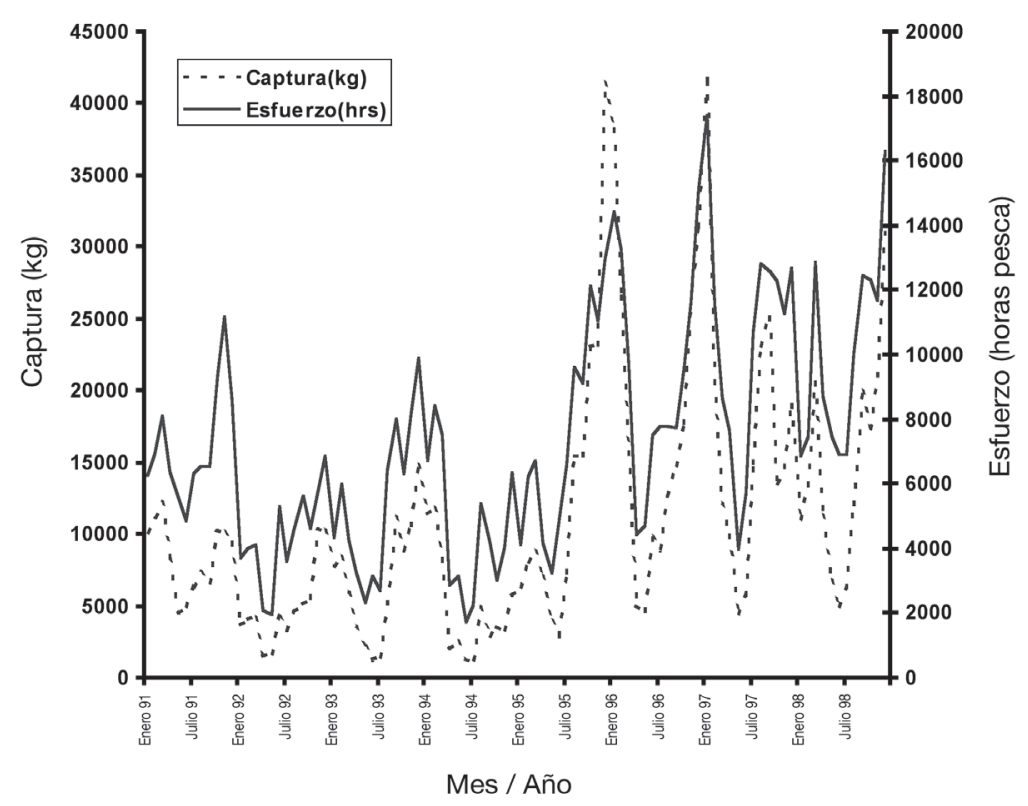

Fig. 5. Variación en los niveles de esfuerzo y captura presentados en la pesquería de arrastre del camarón blanco (L. stylirostrsis y L. occidentalis) en el Golfo de Nicoya., Costa Rica.

Fig. 5. Variation in the effort and capture levels presented in the white shrimp trawl fishery (L. stylirostris and L. occidentalis) in the Gulf of Nicoya, Costa Rica.

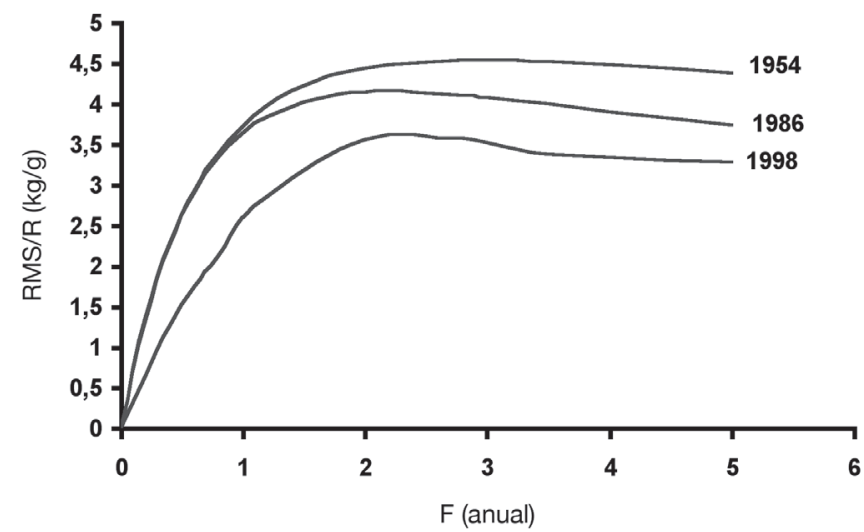

Fig. 6. Efecto del incremento en los niveles de pesca (F) para tres años, sobre la capacidad de renovación de la población del camarón blanco (RMS/R) capturada por la flota de arrastre en el Golfo de Nicoya.

Fig. 6. Effect of the increment in fishing levels (F) for three years, on the renewal capacity (RMS/ R)of the white shrimp population catched by trawl fleet in the Gulf of Nicoya, Costa Rica.

y Fox para la pesquería de arrastre del camarón blanco se presentan en el Cuadro 2. De acuerdo con los datos de captura y esfuerzo, el RMS se alcanzó en la década de los 70 y principios de $\operatorname{los} 80$ (Fig. 7).
Después de 1977 decae la captura, ya los reclutas que incorpora la población desovante son insuficientes para reemplazar a los individuos que sufren mortalidad por pesca, lo que indica que la pesquería del camarón blanco 


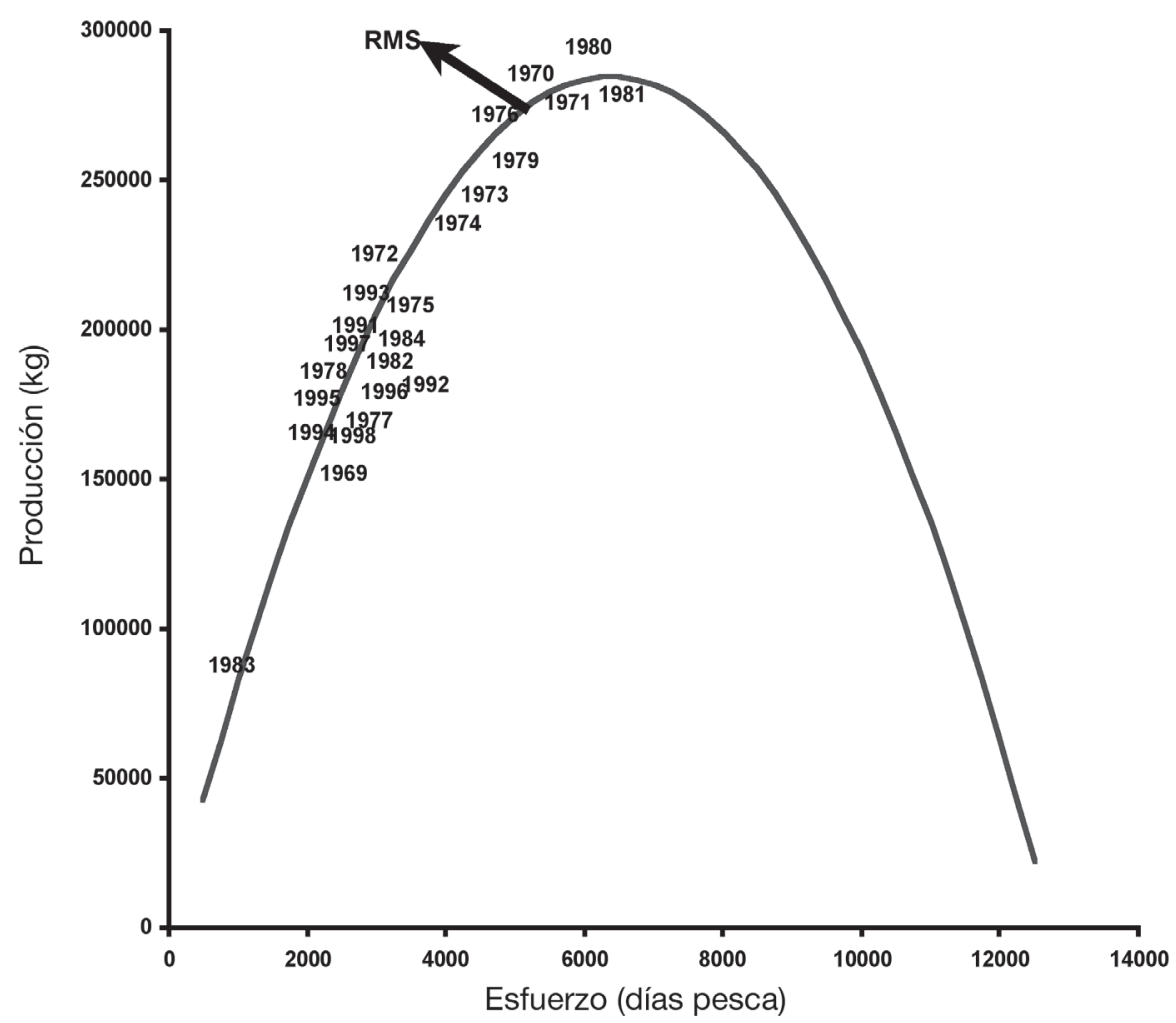

Fig. 7. Evolución histórica de la curva de Schaefer para la estimación del Máximo Rendimiento Sostenible (MRS) en la pesquería de arrastre del camarón blanco, L. occidentalis y L. stylirostris en el Golfo de Nicoya, Costa Rica.

Fig. 7. Historical evolution of the Schaefer curve for the estimation of Maximum Sustainable Yield (MSY) in the white shrimp trawl fishery, L. occidentalis and L. stylirostris, Gulf of Nicoya, Costa Rica.

\section{CUADRO 2}

Determinación del Rendimiento Máximo Sostenido (RMS) $y$ del esfuerzo óptimo en la pesquería de arrastre del camarón blanco (L. stylirostris y L. occidentalis) en el Golfo de Nicoya, Costa Rica

\section{TABLE 2}

Determination of the Maximum Sustainable Yield (MSY) and optimal effort in the white shrimp (L. stylirostris and L. occidentalis) trawl fishery, Gulf of Nicoya, Costa Rica

\begin{tabular}{lcc}
\multicolumn{1}{c}{ Parámetro } & Schaefer & Fox \\
RMS $(\mathrm{Kg})$ & 284726.87 & 282369.97 \\
$\mathrm{f}_{\text {RMS }}$ (días pesca) & 6377 & 6235 \\
$\mathrm{r}^{2}$ & 0.73 & 0.75
\end{tabular}

inicia su ingreso en la fase de sobrepesca por recluta. Para la década de 1980, ya la población es sometida a un esfuerzo tal que la biomasa disminuye drásticamente y en los 90 la población presenta una capacidad nula de renovación natural de la población (Fig. 7)

De acuerdo con este modelo analítico, durante la década del 90, la captura oscila entre 150- 250 ton anuales, aún cuando el esfuerzo ha experimentado cambios de corto plazo de gran magnitud, esto es, la disminución en las capturas en relación con los primeros cinco años de la década de los 70 (que era cuando la población de camarón blanco estaba en equilibrio), es de $71.4 \%$. 


\section{DISCUSIÓN}

El análisis de los datos históricos de producción indican que la pesquería de arrastre del camarón blanco y del rosado alcanzó su pico de producción en 1975 y 1980, respectivamente, y la del fidel probablemente entre 1983 y 1985.

Integralmente la pesquería de arrastre del camarón alcanzó su MRS en períodos muy similares, no obstante que la del camarón blanco inició en 1952, la del camarón rosado en 1956 y la del camarón fidel en 1978. Esto indica que la capacidad de renovación natural del camarón blanco es mucho mayor que la de los otros dos. Pero también, es un indicativo que deja entrever que la pesca del camarón fidel rápidamente alcanzó su MRS, esto sucedió entre 1984 y 1985, lo que indica que probablemente sea la población ecológicamente más frágil, y que desde un principio la población desovante empezó a sufrir directamente las consecuencias de la mortalidad por pesca. Se estima que en términos absolutos, esta población en la actualidad presenta una sobrepesca de un $168 \%$ en relación con las capturas informadas en 1981. Resultados similares se observan en otras pesquerías multiespecíficas de camarón (DFO 1999, Siddeek et al. 2001, Love y Bishop 2002)

Una característica de muchas de las curvas de captura observadas hasta ahora es su tendencia a comportarse como una curva de tipo eumétrica, esto es, los cambios anuales en los niveles de captura están afectando directamente los niveles de reclutamiento en la población del camarón blanco. Evidencia de esta afirmación se observa al analizar los datos de captura y esfuerzo en relación con los estimados de mortalidad (natural y por pesca) y con los análisis de patrones de reclutamiento obtenidos para esta especie por Tabash y Palacios (1996) (Fig. 6).

Obsérvese en la Figura 6 como en 1954 la relación entre el peso por recluta que reponía a los camarones afectados por la mortalidad por pesca era casi de $4.5 \mathrm{~g} / \mathrm{kg}$, en 1986 era de 3.5 $\mathrm{g} / \mathrm{kg}$ y en 1999 descendió a $3 \mathrm{~g} / \mathrm{kg}$. Esto implica que en 1954 más camarones blancos sobrevivían y eran capaces de mantener una población desovante tal que el efecto de mortalidad por pesca era igualado por el de reclutamiento.

La magnitud de los registros de captura de camarón fidel obtenidos en 1998 de 247 ton excede el estimado de MRS de 127 ton obtenido en este análisis y que como ya se afirmó, se alcanzó entre 1984 y 1985. Esto indica que el nivel de sobrepesca en 1998, considerándolo sobre el punto de equilibrio, fue de un $51 \%$.

Para el caso de la pesquería de camarón rosado, que inició cuatro años después de la del camarón blanco, se estima que el MRS fue de $249665.6 \mathrm{~kg}$ (250 ton) que es producto de un esfuerzo óptimo de 2161 días pesca, esta cantidad se alcanzó en 1995, considerando que el análisis se realizó con datos del período comprendido entre 1991 y 1998 . Es muy probable que ya para 1986 la pesquería haya alcanzado el MRS. Esto debido a que a partir de 1995, las capturas disminuyeron de 514 ton en 1994 a 284 ton al año siguiente, o sea una reducción de un $55 \%$. Sin embargo, la reducción del esfuerzo fue solo de un $7 \%$, evidencia de que el efecto sobre la población desovante se manifestó hasta 1995, en otras palabras, esta pesquería probablemente alcanzó el MRS en 1986 y no es hasta 1995 que ingresa en el proceso de sobrepesca por recluta. En 1988, ya el nivel de sobre-explotación estimada era de un $62 \%$ (Tabash y Palacios 1996), lo que respalda la disminución en el RMS y el aumento en la demanda de reclutas de camarón requeridos para tratar de reponer a los que son capturados por la flota arrastrera, en consecuencia la edad de primera captura disminuye exponencialmente.

En el caso de la pesquería del camarón blanco se dispone de una mayor cantidad de datos, producto del trabajo de Alfaro et al. (1993) y Alfaro (1994) en reproducción, Bravo (1978, 1979), Carranza y Chacón (1978), Carranza (1985, 1990), Gutiérrez (1985), Misión Técnica Española (1988), Angulo (1993), Palacios et al. (1993), Chacón (1994), Tabash y Palacios (1996), y Tabash y Chávez (2006), sobre aspectos pesqueros; lo que permitió que el análisis fuera más integral.

Los estimados obtenidos para la aplicación del modelo de Schaefer, establecen la relación 
existente entre los niveles de pesca que se presentaron en la década del 90 y la ubicación del máximo rendimiento en capturas que esta pesquería debería de tener, para que la mortalidad por pesca iguale los patrones de reclutamiento.

El MRS, calculado en $284727 \mathrm{~kg}$ (285 ton) fue alcanzado por esta pesquería entre 1970 y 1976, esto significa que fue en ese período que la población del camarón blanco, integrada en aquel entonces no solo por $L$. occidentalis y L. stylirostris, sino también por L. vannamei (que desaparece de la pesquería a principios de los 80) presentaba una población de jóvenes reclutas tal que permitían que los individuos que eran capturados (mortalidad por pesca) fuesen reemplazados lo que mantenía la población en equilibrio.

$\mathrm{Al}$ incrementar el esfuerzo pesquero se provoca un enrarecimiento del stock que afecta los patrones de reclutamiento exhibidos por la población de camarón blanco. Una densidad demasiado reducida del stock parental dificulta los encuentros entre machos y hembras por lo tanto la fecundación.

Es evidente que la disminución en la relación biomasa de reclutas; RMS, entre 1952 y 1998, ha sido el principal factor que ha hecho que la población del camarón blanco se encuentre en la fase de sobre-explotación biológica del recurso, o lo que es igual de sobre-explotación de reclutas. Este análisis histórico comparativo se presenta como respaldo de que el nivel de sobre-explotación a que ha sido sometido el camarón blanco, está afectando los jóvenes o reclutas.

Por tanto, considerando que toda la población de camarones se encuentra sobreexplotada desde 1976 y que además los análisis de ingreso de reclutas, estiman que la única forma de recuperar la población a mediano plazo es mantener la mortalidad por pesca $(\mathrm{F})$ en 0 , se recomienda que la actividad de pesca de arrastre del camarón, ya sea este blanco, rosado, café o fidel debe cesar por completo en el Golfo de Nicoya.

Se sugiere vedar la zona del Golfo de Nicoya, desde Playa Mantas hasta Puntarenas a la pesca de arrastre del camarón, no solo por el camarón sino por el daño que causa sobre la fauna de acompañamiento. Al tiempo que inicie un proceso de monitoreo que permita saber cuánto tiempo requiere la población para recuperarse, asimismo se presente e implemente un Plan de Ordenamiento del Recurso Camarón en el Golfo de Nicoya.

\section{RESUMEN}

En Costa Rica, la pesquería del camarón que se lleva a cabo en el Golfo de Nicoya desde 1952 representó una de las más importantes actividades económicas de la región. Sin embargo, la sobrepesca redujo los volúmenes de captura al punto de impedir esta actividad comercial. Con el objetivo de evaluar su evolución durante la pasada década y compararla con los registros históricos de captura, se analizaron las estadísticas pesqueras oficiales comprendidas entre 1991 y 1999 para las especies de camarón que se capturan: dos especies de camarón blanco (Litopenaeus occidentalis y L. stylirostris), el camarón tití (Xiphopenaeus riveti), el camarón café (Farfantepenaeus californiensis), el camarón "pinki” (F. brevirostris) y el camarón fidel (Solenocera agassizi). Todas las especies analizadas presentaron un alto nivel de sobre-explotación, lo que provoca un aumento en la demanda de reclutas de camarón requeridos para tratar de reponer a los que son capturados por la flota arrastre. En consecuencia, la edad de primera captura ha disminuido exponencialmente. Se recomienda vedar totalmente la pesca de arrastre del camarón en el Golfo de Nicoya.

Palabras clave: camarón, pesca, Golfo de Nicoya, evaluación, colapso, sobrepesca, veda.

\section{REFERENCIAS}

Alfaro, J. 1994. Ultraestructura de la glándula androgénica. Espermatogénesis y oogénesis de camarones marinos (Decapoda: Penaeidae). Rev. Biol. Trop. 42: 121129.

Alfaro, J., J.A. Palacios, T. Aldave \& R. Angulo. 1993. Reproducción del camarón Penaeus occidentalis (Decapoda: Penaeidae) en el Golfo de Nicoya, Costa Rica. Rev. Biol. Trop. 41: 563-572.

Angulo, R. 1993. Crecimiento, mortalidad y madurez sexual de Penaeus stylirostris, Penaeus occidentalis y Trachipenaeus byrdi en el Golfo de Nicoya, Costa Rica. Tesis de Licenciatura, Universidad Nacional, Heredia, Costa Rica. 73 p. 
Bravo, E. 1978. Análisis sobre la pesca del camarón y sardina en la zona comprendida entre Negritos-Peñón y PuntarenasPunta Gigante, período 1977. Mimeografiado. MAG: Dirección de Recursos Pesqueros y Vida Silvestre, San José, Costa Rica. 47 p.

Bravo, E. 1979. Características generales de los recursos camaroneros de Costa Rica. MAG: Dirección General de Recursos Pesqueros y Vida Silvestre, San José, Costa Rica. 27 p.

Carranza, F. 1985. Distribución y abundancia del recurso camarón blanco y alternativas de aprovechamiento mediante un "método de pesca artesanal" en el Golfo de Nicoya. Tesis de Maestría, UNAM, México DF, México. 60 p.

Carranza, F. 1990. La veda para la pesca con redes de enmalle (trasmallos) en el Golfo de Nicoya y para la pesca de camarón blanco, café, tití en el litoral pacífico costarricense. Mimeografiado. Oficina de Pesca-MAG, San José, Costa Rica. 64 p.

Carranza, F. \& A. Chacón. 1978. Estudios preliminares sobre la pesca del camarón blanco, café y rosado del litoral pacífico costarricense. Oficina de Pesca-MAG, San José, Costa Rica. 45 p.

Chacón, A. 1994. Algunos aspectos relevantes de la pesquería costarricense. Mimeografiado. GUTEPLA, San José, Costa Rica. 11p.

Departament of Fisheries and Oceans (DFO). 1999. Shrimp trawl fishery of the west coast of Canada. DFO Science Stock Status Inform., Montreal, Canadá. $21 \mathrm{p}$.

Fox, W.W. 1970. An exponential surplus-yield model for optimizing exploited fish populations. Trans. Am. Fish. Soc. 99: 80-88.

Gulland, J.A. 1983. Fish stock assessments: A manual of basic methods. Chichester, U.K., Willey Interscience, FAO/Willey series on Food and Agriculture 1: 223 p.

Gutiérrez, R. 1985. Producción camaronera durante los últimos 15 años en el litoral pacífico de Costa Rica. In Mem. 1er. Sem. sobre la problemática pesquera en Costa Rica, 4-7 Dic. INCOPESCA Puntarenas, Costa Rica. 9 p.

Herrington, W.C. 1943. Some methods of fishery management and their usefulness in a management program. U.S. Fish and Wild. Serv., Spe. Sci. Rep.18: 3-22.

Instituto Costarricense de Pesca y Acuacultura (INCOPESCA). 1999. Boletines anuales de Pesca. Departamento de Estadística-INCOPESCA, San José, Costa Rica. 39 p.
Love, D. \& G. Bishop. 2002. Inform to the board of fisheries Southeast Alaska shrimp beam trawl fisheries. Regional Information Inform No. 1302-45. Alaska Dept. of Fish and Game, Juneau, Alaska, EEUU. 24 p.

López, G.L. 1968. Estudio preliminar sobre las migraciones de postmisis de Penaeus vannamei Boone, p. 405-413. In M.N. Mistakidis (ed.). Actas de la Conf. Cient. Mundial sobre Biol. y Cultivo de camarones y gambas, México DF, 12-21 de junio. Vol. 2. Resumen regional y Docs. de investigación. FAO. Fish. Rep. 57: 577-587.

Martínez, F. 1988. La pesquería del camarón en el litoral pacífico costarricense: Misión Técnica Española II. Centro regional de Pesca, Puntarenas, Costa Rica. $250 \mathrm{p}$.

Misión Técnica Española. 1988. La pesquería del camarón en Costa Rica: Análisis del camarón blanco. San José, Costa Rica. 39 p.

Mostella, F. \& J.W. Tukey. 1977. Data analysis and regression: A second course in statistics. Addison-Wesley Red, Boston, Massachusetts, EEUU. 167 p.

Palacios, J.A. 2003. Estado actual de los recursos pesqueros del litoral pacífico de Costa Rica: Cadena de productos hidrobiológicos, compromiso del sector pesca. Manuscrito. Estación de Biología Marina, Universidad Nacional, Puntarenas, Costa Rica. 114p.

Palacios, J.A., J.A. Rodríguez \& R. Angulo. 1993. Algunos aspectos biológico-pesqueros para la ordenación de la pesquería del camarón blanco (Penaeus stylirostris) en el Golfo de Nicoya, costa Rica, p 119-126. In Actas del Simp. Inv. Acuic. y Pesca en C.A.

Pauly, D. 1984. Fish population dynamics in tropical waters: A manual for use with programmable calculators. ICLARM, Stud. Rev. 8: 325.

Ricker, W.E. 1975. Computation and interpretation of biological statistics of fish populations. Bull. Fish. Res. Board Can. 191: 382.

Schaefer, M.B. 1954. Some aspects of the dynamics of populations important to the management of the commercial marine fisheries. Inter-Am. Trop. Tuna. Comm. Bull. 1: 27-56.

Schaefer, M.B. 1957. A study of the dynamics of the fishery for yellowfin tuna in the eastern tropical Pacific ocean. Inter-Am. Trop. Tuna. Comm. Bull. 2: $247-268$.

Siddeek, M.S.M., G. Hermosa, M.N. Al-Amri \& A. AlAisery. 2001. Stock assessment of shrimp in the Gulf of Masirah, Sultanate of Oman, p. 107-118. In S. 
Goddard., H. Al-Oufi, J. McIlwain \& M. Claereboudt (eds.). Proc. 1st International Conference on Fisheries, Aquaculture and Environment in the NW Indian Ocean. Sultan Qaboos University, Muscat, Omán.

Sparre, P. \& S.C. Venema. 1992. Introduction to tropical fish stock assessment. Part I. Manual FAO Fish.Tech. Pap. No. 306.1. Rev. 1. FAO, Roma, Italia. 376 p.

Tabash, B.F. \& E.A. Chávez. 2006. Optimizing harvesting strategies of the white shrimp fishery in the Gulf of Nicoya, Costa Rica. Crustaceana 79: 327-343.
Tabash, B.F. \& J.A. Palacios. 1996. Stock assessment of two penaeid prawn species, Penaeus occidentalis and Penaeus stylirostris (Decapoda: Penaeidae) in Golfo de Nicoya, Costa Rica. Rev. Biol. Trop. 44: 595-602.

Tukey, J.W. 1977. Exploratory data analysis. AddisonWesle, Reading, Massachusetts, EEUU. 18 p.

Vitola, M.M. 1985. Camarones penaeidos (Decapoda: Natantia) el Golfo de Nicoya, Costa Rica: Un análisis de su distribución y densidad. Tesis de Maestría, Universidad de Costa Rica, San José, Costa Rica. 53 p. 